\title{
An overview of genetically modified crop governance, issues and challenges in Malaysia
}

\begin{abstract}
The application of agricultural biotechnology attracts the interest of many stakeholders. Genetically modified (GM) crops, for example, have been rapidly increasing in production for the last 20 years. Despite their known benefits, GM crops also pose many concerns not only to human and animal health but also to the environment. Malaysia, in general, allows the use of GM technology applications but it has to come with precautionary and safety measures consistent with the international obligations and domestic legal frameworks. This paper provides an overview of GM crop technology from international and national context and explores the governance and issues surrounding this technology application in Malaysia. Basically, GM research activities in Malaysia are still at an early stage of research and development and most of the GM crops approved for release are limited for food, feed and processing purposes. Even though Malaysia has not planted any GM crops commercially, actions toward such a direction seem promising. Several issues concerning GM crops as discussed in this paper will become more complex as the number of GM crops and varieties commercialised globally increase and Malaysia starts to plant GM crops.
\end{abstract}

Keyword: Malaysia; Biosafety; Biotechnology; Genetically modified crop; Genetically modified organism 